with $\bar{A}(0)=A(0)$, in comparison with Eq. (20), it may be understood that a sort of Langevin equation including fluctuating term appears after removing the projection operator in Eq. (25) to furnish with a kind of causality.

Finally, it may be shown that a generalized relationship of Einstein and Nernst is established as an Abel limit of Eq. (14) with Eq. (15).

In the limit of $s \rightarrow+0$ of Eq. (14), the following equation is obtained:

$$
\begin{aligned}
\lim _{s \rightarrow+0} \int_{0}^{\infty} d t \exp & (-s t)\left\{\operatorname{Tr} \varrho A^{+}(0) A(t)\right\} \\
& =\int_{0}^{\infty} d t \exp (-t / \tau)\left\{\operatorname{Tr} \varrho A^{+}(0) A(0)\right\}
\end{aligned}
$$

where

$$
\tau=\left\{-i P \omega^{*}+\zeta_{s}\right\}^{-1}
$$

$\zeta_{s t}$ is nothing but a friction coefficient in a stationary Markoffian process, and

$$
\zeta_{s t}=\lim _{s \rightarrow+0} \int_{0}^{\infty} d t \exp (-s t)\left\{\operatorname{Tr} \varrho \widetilde{F}^{+}(0) \mathfrak{F}(t)\right\}
$$

Because of

$\times\left(\operatorname{Tr} \varrho A^{+} A\right)^{-1}$

$$
-i P \omega^{*}=\left(\operatorname{Tr} \varrho A^{+} A\right)^{-1} \cdot\left\{\operatorname{Tr} \varrho A^{+}(0) A(0)\right\},
$$

Eq. (26) becomes a generalized Einstein-Nernst relation of

$$
\begin{aligned}
& \left(\operatorname{Tr} \varrho A^{+} A\right) / \tau=\operatorname{Tr} \varrho \mathfrak{F}^{+}(0) A(0) \\
& \quad+\lim _{s \rightarrow+0} \int_{0}^{\infty} d t \exp (-s t)\left\{\operatorname{Tr} \varrho \mathfrak{F}^{+}(0) \tilde{\delta}(t)\right\} .
\end{aligned}
$$

For example, according to Langevin's formalism, the equation of motion of a Brownian particle, whose mass is $m$, the momentum $p$, is written as

$$
d p / d t=-\zeta(p / m)+\mathfrak{F}(t),
$$

where the property of dissipation is due to the first term on the right-hand side, and the second term is a fluctuating force. Because of the momentum relaxation time $\tau_{p}=m / \xi$, the Einstein-Nernst relation leads to

$$
\begin{aligned}
\left(\operatorname{Tr} \varrho p^{2}\right) / \tau_{p}=m k T / \tau_{p} & \\
& =\lim _{s \rightarrow 0} \int_{0}^{\infty} d t \exp (-s t)\{\operatorname{Tr} \varrho \mathfrak{F}(0) \mathfrak{F}(t)\},
\end{aligned}
$$

which is usually written as

$$
m / x_{p}=\zeta=(1 / k T) \int_{0}^{\infty} d t\{T r \varrho \mathfrak{F}(0) \mathfrak{F}(t)\} .
$$

Here, it is evident that the phase-space distribution function in equilibrium has been used in place of quantum density matrix, and also an integral referring to the elementary volume of the phase space was taken instead of a trace of a matrix, $T r$.

In this article, we have derived several equations having wide ranges of validity and applicability, from Heisenberg's equation of motion in quantum mechanics with Zwanzig's technique of projection operator ${ }^{5}$.

Zusammenfassung. Die Gleichung von Langevin wurde mit Hilfe der $Z$ wanzigschen Methode von Projektionsoperator aus der quantenmechanischen Bewegungsgleichung von Heisenberg geführt, die allgemein als Grundgleichung der Brownschen Bewegung gilt. Zum Derivieren der Gleichung, ist es notwendig, eine Definition für Projektionsoperator $P$ mit einem statistischen Operator $\varrho$ aufzunehmen, das ist:

$$
\begin{aligned}
P A(t) & \equiv(A(0), \quad \varrho A(0))^{-1} \cdot(A(0), \varrho A(t)) A(0) \\
& =\left\{\text { Spur } \varrho A^{+}(0) A(0)\right\}^{-1} \cdot\left\{\operatorname{Spur} \varrho A^{+}(0) A(t)\right\} A(0) \\
& =\Xi(t) A(0)
\end{aligned}
$$

wobei $\left(A^{\prime}, A^{\prime \prime}\right)$ ein inneres Produkt zwischen zwei Vektoren $A^{\prime}$ und $A^{\prime \prime}$ ist. Die Zeitveränderlichkeit einer Observablen $A \mathrm{im}$ Heisenberg-Bild gegeben ist:

$$
\begin{aligned}
& A(t)=\exp (i t H / \hbar) A(0) \exp (-i t h H / \hbar) ; \operatorname{mit} \hbar=h / 2 \pi, \\
& \Xi(t)=\left\{\operatorname{Spur} \varrho A^{+}(0) A(0)\right\}^{-1} \cdot\left\{\text { Spur } \varrho A^{+}(0) A(t)\right\},
\end{aligned}
$$$$
A^{+} \text {zeigt den adjungierten Operator von } A \text {, und }
$$

$$
(i / \hbar)[(1-P) H, \quad \Xi(t)]=0 .
$$

Hierauf $(1-P)$ ist der orthogonalkomplementale Projektionsoperator zu $P$.

\section{N. TAKEYAMA}

Department of Applied Chemistry, Faculty of Engineering, Kyushu University, Fukuoka (Japan), May 22, 1966.
5 The main contents of this article were published in Japanese in Bussei Kenkyu 5, 275 (1966).

- The author would like to express his sincere thanks to Professor T. SeIYama for his encouragement.

\title{
CORRIGENDA
}

C. Paradisi: Mitochondrial Changes Induced by Diphtheria Toxin in Chicken Embryo Heart Cell Cultures, Experientia 22, fasc. 6, p. 373 (1966). On page 373, line 15 reads correctly as follows: 'This toxin induces a clear swelling in isolated mitochondria'. The author points out that Dr. Kadis has never stated or implied that murine plaque toxin causes uncoupling of oxidative phosphorylation.
S. Gelfand and A. Ganz: Further Observations of the Angiotensin Vasopressive Effect in Rabbits, Experientia 22, fasc. 7, p. 478 (1966). The author has noted that the dosage of angiotensin should read $\boldsymbol{\mu} \mathrm{g}$ instead of $\mathrm{mg}$. 